\title{
PRODUCCIÓN DE POLEN EN UN MATORRAL DEL SUR DE ESPAÑA
}

\author{
María Isabel HIDALGO, Marta RECIO y Baltasar CABEZUDO
}

\begin{abstract}
RESUMEN. Producción de polen en un matorral del Sur de España. Se ha realizado una estimación de la producción de polen (en número de granos y en volumen) de 61 especies de un matorral del Sur de España. La determinación se ha realizado por flor y por unidad de superficie de planta y año. También se ha estimado la importancia polinífera (IP) que cada especie tiene en el matorral en función de su cobertura. La especie que ha presentado mayor número de granos de polen por flor y metro cuadrado y año es Myrtus communis. El máximo valor de volumen de polen por flor lo tiene Cistus albidus. El mayor volumen de polen por metro cuadrado y año corresponde a Lavandula stoechas. Las especies de mayor IP en la zona de estudio han sido Lavandula stoechas, Rosmarinus officinalis, Cistus albidus, Quercus coccifera, Quercus rotundifolia, Cistus clusii, Thymbra capitata, Ulex baeticus y Cistus monspeliensis. En la zona estudiada hay un elevado número de especies que presentan valores significativamente altos en su producción de polen por superficie y año. El polen que produce la Sierra de Mijas es un buen recurso alimenticio para la abeja doméstica Apis mellifera durante todo el año.
\end{abstract}

Palabras clave. Polen, producción, recurso apícola, Apis mellifera, Andalucía, España.

ABSTRACT. Pollen production in a scrub of Southern Spain. We estimated the pollen production (in volume and grain number) of 61 species of a scrub in southern Spain. The calculation was made per flower and per plant area and year. In addition, the polliniferous importance (PI) of each species as a function of its cover was estimated. The species producing the greatest number of pollen grains per flower and per square metre and year was Myrtus communis, while the maximum pollen volume per flower was observed in Cistus albidus. The greatest volume of pollen per square metre and year was produced by Lavandula stoechas. The highest PI in the area studied correspond to Lavandula stoechas, Rosmarinus officinalis, Cistus albidus, Quercus coccifera, Quercus rotundifolia, Cistus clusii, Thymbra capitata, Ulex baeticus and Cistus monspeliensis. Many species showed significant high values of pollen production per area and year. The pollen produced in the Sierra de Mijas is a good source of food for the domesticated bee, Apis mellifera, throughout the year.

Key words. Pollen production, apicultural resource, Apis mellifera, Andalusia, Spain.

\section{INTRODUCCIÓN}

La cuantificación del polen que producen las flores de una determinada especie resulta de gran interés en los estudios melitopalinológicos ya que, junto con el néctar, es uno de los recursos alimenticios que más comunmente utiliza la abeja doméstica (Apis mellifera). La mayoría de los trabajos de palinología aplicados a la apicultura se han 
realizado en las dos últimas décadas, destacando los realizados en la zona mediterránea por Ricci Ardeli \& Vorwohl (1979), Weber El-Ghobary (1984) y Bolchi (1986-87); y en Andalucía (España) por Herrera (1985), Devesa et al. (1985), Ortiz (1985, 1988, 1991, 1994), Talavera et al. (1988), y Ortiz et al. (1990).

La productividad polínica de una especie en una zona determinada depende, en definitiva, de tres factores: cantidad de polen por flor, cantidad de flores por unidad de superficie a lo largo de un periodo completo de floración, e importancia de la especie en el conjunto de la vegetación de la zona estudiada. Mientras que los dos primeros caracteres son propios de cada especie, el tercero es muy variable en función de la cobertura y dominancia que la especie presenta en la formación vegetal estudiada, por lo que es el factor responsable de la importancia real de cada especie en productividad polínica total. De acuerdo con Ortiz (199l), también debe tenerse en cuenta el tamaño del grano de polen a la hora de cuantificar la producción por flor en relación con la alimentación de los insectos. Una especie puede producir gran cantidad de polen, pero si éste es de pequeño tamaño, la oferta alimenticia resultante (en peso o volumen) no va a resultar tan grande. Este hecho fue inicialmente puesto de manifiesto por Louveaux (1958) en lo referente a la intervención de cada especie en las cargas polínicas.

El objetivo de este trabajo es determinar la producción de polen por flor, en número de granos y volumen, de las especies más importantes del matorral típico (aulagar-jaral) de la Sierra de Mijas (Málaga), y estimar su producción total en función de la cobertura y producción floral de cada especie en la zona de estudio (Hidalgo et al. 1994, 1996). Estos resultados nos permitiran evaluar la importancia que cada especie tiene como recurso alimenticio de Apis mellifera.

\section{METODOLOGÍA}

La Sierra de Mijas, situada en la provincia de Málaga, entre los $4^{\circ} 43^{\prime}$ y $4^{\circ} 30^{\prime}$ de longitud Oeste y los $36^{\circ} 33^{\prime}$ y $36^{\circ} 40^{\prime}$ de latitud Norte, se caracteriza por presentar un clima de tipo mediterráneo, con temperaturas y precipitaciones medias anuales de $17,5^{\circ} \mathrm{C}$ y $610 \mathrm{~mm}$ respectivamente. Las temperaturas máximas se producen en Agosto $\left(25,6^{\circ} \mathrm{C}\right.$ de media) y las mínimas en Enero $\left(11,1^{\circ} \mathrm{C}\right.$ de media). Las lluvias se producen fundamentalmente en otoño e invierno, y desde finales de Mayo hasta finales de Septiembre es característico un periodo de aridez y máxima insolación.

Biogeográficamente, la zona de estudio pertenece a la Región Mediterránea y se incluye en el Subsector Mijense del Sector Rondeño (Provincia Bética). La vegetación está representada por un coscojar-encinar perteneciente a la asociación Rhamno velutiniQuercetum cocciferae, comunidad formada por fanerófitos y nanofanerófitos que colonizan suelos sobre mármoles dolomíticos. En zonas más degradadas domina un matorral de jaras y aulagas perteneciente a la asociación Cisto clusii-Ulicetum baetici. Otros tipos de comunidades, importantes florísticamente, son las que se asientan sobre pedregales, arenales y cunetas, constituídas por numerosos hemicriptófitos y terófitos característicos del Sector Rondeño (Nieto et al., 1988, 1991).

La cuantificación de polen se ha realizado en 61 especies, consideradas como las de mayor interés polinífero en la zona. Para estimar el número de granos de polen por flor se ha seguido básicamente el método propuesto por Cruden (1977). Para ello se recolectaron botones florales de varios individuos de cada una de las especies y se fijaron en alcohol al $70 \%$ hasta el momento de su análisis. Bajo una lupa binocular, se contabilizó el número de anteras de cada flor, tomándose posteriormente, dependiendo del tamaño, una o varias anteras 
que no estuviesen abiertas. En el caso de heteromorfia, bien en las anteras o en los estambres, e incluso cuando los estambres se insertan de distinta manera en una misma flor, se han cogido varias anteras de cada tipo. En cada especie, se ha estimado la producción de polen correspondiente a 5 flores de distintos individuos. Las anteras seleccionadas se han macerado en un tubo de ensayo, donde se ha añadido un volumen conocido de agua destilada con unas gotas de detergente y una pequeñísima cantidad de colorante (zafranina). De la suspensión, bien homogeneizada, se han extraído $5 \mu \mathrm{l}$ que se han depositado sobre un portaobjetos. Se han realizado diez extracciones por tubo, en cada una de las cuales se ha contabilizado el número de granos de polen al microscopio óptico y se ha calculado finalmente la media aritmética de las diez mediciones efectuadas. Posteriormente, conocido el volumen de la suspensión y el número de anteras totales que presenta una flor de una especie, multiplicando se obtiene el total de granos de polen de cada flor. Finalmente se ha calculado la media aritmética y la desviación estándar, obteniéndose así una estimación del número de granos de polen en una flor de una especie.

La producción en volumen de polen de cada especie se ha estimado multiplicando la media del número de granos de polen por flor por el volumen medio que presenta cada grano de polen, que viene dado en función de los ejes principales del grano de polen ( $\mathrm{P}$ y E). La producción (en número y en volumen) de polen por $\mathrm{m}^{2}$ de cada especie $\left(\mathrm{n}^{\circ} \mathrm{gP} / \mathrm{S}\right.$ y $\mathrm{VgP} / \mathrm{S}$, respectivamente) se ha calculado en función del número medio de flores que produce dicha especie por unidad de superficie y fenofase floral completa (Hidalgo et al., 1996). Finalmente, con objeto de obtener una estimación de la importancia polinífera (IP) que cada especie tiene en la zona de estudio, se ha multiplicado su volumen de grano de polen por superficie y año $(\mathrm{VgP} / \mathrm{S})$ por su porcentaje de cobertura (M\%) en el matorral estudiado (Hidalgo et al., 1994); el resultado nos da un índice (IP) que indica una jerarquización de especies en función de la producción de polen en ese matorral concreto, y que puede ser aplicado a todos los matorrales ecológicamente semejantes al estudiado (matorrales basófilos, termófilos y subhúmedos de las asociaciones Rhamno velutini-Quercetum cocciferae y Cisto clusii-Ulicetum baetici).

\section{RESULTADOS Y DISCUSIÓN}

El número medio de granos de polen por flor (tab. 1, $\mathrm{n}^{\circ} \mathrm{gP} /$ flor) oscila entre los 1.044 .800 de Myrtus communis y los 1.571 de Helichrisum stoechas, destacando además por su alta producción Cistus albidus (391.100), Cistus crispus (391.110), Cytinus hypocistis (673.955), Reseda suffruticosa (396.813) y Ranunculus gramineus (232.280). Los valores de $n^{\circ} \mathrm{gP} /$ flor obtenidos en las diferentes especies estudiadas se aproximan a los obtenidos por Ortiz (1991) y Talavera et al. (1988) en Andalucía Occidental. Se han observado grandes desviaciones estándars, debidas fundamentalmente a la variabilidad dentro de los individuos de la misma especie, los diferentes estados de desarrollo de la planta, y al método de conteo.

Referente al número de granos de polen por $\mathrm{m}^{2}$ y fenofase completa (tab. $1, \mathrm{n}^{\circ} \mathrm{gP} / \mathrm{S}$ ) se

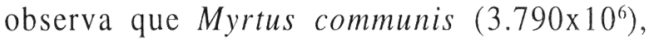
Ferula communis $\left(3.563 \times 10^{6}\right)$, Quercus rotundifolia $\left(3.206 \times 10^{6}\right)$ y Echium plantagineum $\left(2.716 \times 10^{6}\right)$ son las especies más productivas; Myrtus communis fundamentalmente por su elevado $\mathrm{n}^{\circ} \mathrm{gP} /$ flor $\mathrm{y}$ las restantes por su alta producción anual de flores por $\mathrm{m}^{2}$ (Hidalgo et al., 1996). En los casos de Cistus albidus y Cistus crispus, que producen número de granos de polen por flor muy similares, se observa que Cistus albidus produce más por $\mathrm{m}^{2}$ que $C$. crispus debido a su 


\begin{tabular}{|c|c|c|c|c|c|}
\hline Familias/Especies & $n^{0}$ gP/flor & $n^{0} \mathrm{gP} / \mathrm{S}\left(\mathrm{x} \mathbf{1 0}^{6}\right)$ & VgP/flor & $\mathrm{VgP} / \mathrm{S}$ & IP (M\%) \\
\hline $\begin{array}{l}\text { AMARYLLIDACEAE } \\
\text { Leucojum autumnale }\end{array}$ & $9.378 \pm 5.400$ & 4,65 & $0,06 \pm 0.03$ & 0,03 & $<0,01 \quad(<0,04)$ \\
\hline $\begin{array}{l}\text { APIACEAE } \\
\text { Ferula communis } \\
\text { Thapsia villosa }\end{array}$ & $\begin{array}{l}19.845 \pm 6.852 \\
10.036 \pm 1.079\end{array}$ & $\begin{array}{c}3.563,98 \\
136,28\end{array}$ & $\begin{array}{l}0,14 \pm 0,04 \\
0,13 \pm 0,01\end{array}$ & $\begin{array}{l}6,28 \\
0,88\end{array}$ & $\begin{array}{l}0,96(0,16) \\
0,38(0,45)\end{array}$ \\
\hline $\begin{array}{l}\text { ARECACEAE } \\
\text { Chamaerops humilis }\end{array}$ & $163.477 \pm 849$ & 147,13 & $2,04 \pm 0,26$ & 1,84 & $2,61(1,41)$ \\
\hline $\begin{array}{l}\text { ASTERACEAE } \\
\text { Calendula arvensis } \\
\text { Carlina corymbosa } \\
\text { Carthamus arborescens } \\
\text { Dittrichia viscosa } \\
\text { Galactites tomentosa } \\
\text { Helichrysum stoechas } \\
\text { Klasea flavescens } \\
\text { Ptilostemon hispanicus }\end{array}$ & $\begin{array}{c}2.721 \pm 934 \\
3.044 \pm 1.149 \\
18.906 \pm 611 \\
3.583 \pm 753 \\
8.155 \pm 1.211 \\
1.571 \pm 315 \\
11.044 \pm 1.581 \\
14.533 \pm 1.877\end{array}$ & $\begin{array}{c}6,63 \\
6,39 \\
59,29 \\
295,66 \\
310,83 \\
437,49 \\
331,32 \\
413,1\end{array}$ & $\begin{array}{c}0,1 \pm 0,08 \\
0,06 \pm 0,02 \\
1,84 \pm 0,06 \\
0,05 \pm 0,01 \\
0,36 \pm 0,05 \\
0,01 \pm 0,002 \\
0,08 \pm 0,005 \\
0,77 \pm 0,09\end{array}$ & $\begin{array}{c}0,24 \\
0,14 \\
5,79 \\
3,87 \\
13,75 \\
3,48 \\
2,58 \\
21,93\end{array}$ & $\begin{array}{c}<0,01(<0,04) \\
0,04(0,25) \\
<0,01(<0,04) \\
0,10(<0,04) \\
0,02(<0,04) \\
0,88(0,25) \\
0,08(<0,04) \\
4,67(0,21)\end{array}$ \\
\hline $\begin{array}{l}\text { BORAGINACEAE } \\
\text { Echium albicans } \\
\text { Echium plantagineum }\end{array}$ & $\begin{array}{c}123.331 \pm 32.121 \\
97.571 \pm 21.456\end{array}$ & $\begin{array}{l}1.805,44 \\
2.716,86\end{array}$ & $\begin{array}{l}0,27 \pm 0,07 \\
0,31 \pm 0,06\end{array}$ & $\begin{array}{l}3,95 \\
8,63\end{array}$ & $\begin{array}{c}1,29(0,32) \\
0,01(<0,04)\end{array}$ \\
\hline $\begin{array}{l}\text { BRASSICACEAE } \\
\text { Diplotaxis virgata }\end{array}$ & $52.600 \pm 2.358$ & 287,09 & $0,71 \pm 0,03$ & 3,87 & $<0,01 \quad(<0,04)$ \\
\hline $\begin{array}{l}\text { CAESALPINIACEAE } \\
\text { Ceratonia siliqua }(\mathrm{m})\end{array}$ & $125.111 \pm 25.027$ & $2.746,56$ & $1,68 \pm 0,33$ & 18,44 & $8,07(0,44)$ \\
\hline $\begin{array}{l}\text { CISTACEAE } \\
\text { Cistus albidus } \\
\text { Cistus clusii } \\
\text { Cistus crispus } \\
\text { Cistus monspeliensis } \\
\text { Cistus salvifolius } \\
\text { Fumana thy mifolia } \\
\text { Halimium atriplicifolium } \\
\text { Halimium halimifolium } \\
\text { Helianthemum cinereum } \\
\text { Helianthemum croceum } \\
\text { Helianthemum syriacum }\end{array}$ & $\begin{array}{c}391.100 \pm 184.813 \\
61.253 \pm 9.384 \\
391.110 \pm 7.698 \\
31.888 \pm 8.335 \\
141.657 \pm 28.295 \\
2.100 \pm 1.273 \\
104.016 \pm 20.799 \\
88.444 \pm 33.048 \\
5.891 \pm 15.948 \\
57.333 \pm 9.428 \\
19.333 \pm 2.357\end{array}$ & $\begin{array}{l}424,34 \\
201,34 \\
305,84 \\
103,06 \\
118,28 \\
22,03 \\
138,55 \\
134,43 \\
22,54 \\
307,93 \\
181,07\end{array}$ & $\begin{array}{l}16,34 \pm 7,72 \\
1,91 \pm 0,29 \\
8,53 \pm 0,16 \\
2,59 \pm 0.67 \\
7,19 \pm 1,43 \\
0,13 \pm 0,08 \\
5,52 \pm 1,06 \\
2,83 \pm 1,06 \\
0,20 \pm 0,05 \\
1,90 \pm 0,31 \\
1,21 \pm 0,10\end{array}$ & $\begin{array}{l}17,73 \\
6,28 \\
6,67 \\
8,37 \\
6 \\
1,36 \\
7,35 \\
4,3 \\
7,65 \\
10,2 \\
11,33\end{array}$ & $\begin{array}{c}107,06(6,02) \\
22,59(3,59) \\
0,20(<0,04) \\
11,90(1,42) \\
0,18(<0,04) \\
0,20(0,15) \\
<0,01(<0,04) \\
1,56(0,36) \\
0,43(0,05) \\
5,56(0,54) \\
1,44(0,13)\end{array}$ \\
\hline $\begin{array}{l}\text { CONVOLVULACEAE } \\
\text { Convolvulus lanuginosus }\end{array}$ & $12.024 \pm 2.225$ & 58,71 & $1,73 \pm 0,32$ & 8,37 & $0,12(<0,04)$ \\
\hline $\begin{array}{l}\text { FABACEAE } \\
\text { Anthyllis cytisoides } \\
\text { Calicotome villosa } \\
\text { Coronilla juncea } \\
\text { Ononis natrix } \\
\text { Psoralea bituminosa } \\
\text { Ulex baeticus }\end{array}$ & $\begin{array}{c}3.755 \pm 63 \\
20.833 \pm 13.531 \\
23.500 \pm 3.330 \\
63.555 \pm 30.939 \\
5.444 \pm 1.171 \\
23.821 \pm 10.940\end{array}$ & $\begin{array}{c}12,58 \\
98,04 \\
97,1 \\
199,37 \\
8,24 \\
281,09\end{array}$ & $\begin{array}{c}0,08 \pm 0 \\
0,17 \pm 0,10 \\
0,08 \pm 0 \\
0,18 \pm 0,09 \\
0,34 \pm 0,07 \\
0,17 \pm 0,08\end{array}$ & $\begin{array}{l}2,57 \\
0,8 \\
0,33 \\
0,56 \\
0,51 \\
2,01\end{array}$ & $\begin{array}{r}1,26(0,48) \\
0,24(0,30) \\
<0,01(<0,04) \\
0,03(0,06) \\
<0,01(<0,04) \\
15,44(7,96)\end{array}$ \\
\hline $\begin{array}{l}\text { FAGACEAE } \\
\text { Quercus coccifera }(\mathrm{m}) \\
\text { Quercus rotundifolia }(\mathrm{m})\end{array}$ & $\begin{array}{l}26.814 \pm 6.479 \\
17.288 \pm 2.701\end{array}$ & $\begin{array}{c}715,26 \\
3.206,92\end{array}$ & $\begin{array}{l}0,25 \pm 0,06 \\
0,17 \pm 0,02\end{array}$ & $\begin{array}{l}42,09 \\
24,26\end{array}$ & $\begin{array}{l}74,17(1,76) \\
67,21(2,77)\end{array}$ \\
\hline $\begin{array}{l}\text { LAMIACEAE } \\
\text { Lavandula stoechas } \\
\text { Rosmarinus officinalis }\end{array}$ & $\begin{array}{c}3.666 \pm 768 \\
8.310 \pm 1.667\end{array}$ & $\begin{array}{l}419,66 \\
261,78\end{array}$ & $\begin{array}{l}0,08 \pm 0,01 \\
0,42 \pm 0,08\end{array}$ & $\begin{array}{c}125,46 \\
13,23\end{array}$ & $\begin{array}{l}163,92(1,30) \\
114,29(8,62)\end{array}$ \\
\hline
\end{tabular}




\begin{tabular}{|c|c|c|c|c|c|}
\hline Familias/Especies & $\mathrm{n}^{\circ} \mathrm{gP} / \mathrm{flor}$ & $\mathrm{n}^{\circ} \mathrm{gP} / \mathrm{S}\left(\mathrm{x} 10^{6}\right)$ & VgP/flor & $\mathrm{VgP} / \mathrm{S}$ & IP $(\mathrm{M} \%)$ \\
\hline Salvia verbenaca & $27.555 \pm 2.341$ & 258,33 & $1,43 \pm 0,12$ & 13,4 & $0,02(<0,04)$ \\
\hline Teucrium fruticans & $13.999 \pm 5.248$ & 26,44 & $0,52 \pm 0,19$ & 0,98 & $0,45(0,46)$ \\
\hline Teucrium lusitanicum & $4.132 \pm 832$ & 300,92 & $0,09 \pm 0,01$ & 6,55 & $4,04(0,61)$ \\
\hline Thymbra capitata & $7.710 \pm 1.452$ & 252,02 & $0,18 \pm 0,03$ & 5,88 & $18,72(3,18)$ \\
\hline Thymus mastichina & $4.321 \pm 1.068$ & 155,33 & $0,11 \pm 0,03$ & 1,97 & $1,48(0,75)$ \\
\hline \multicolumn{6}{|l|}{ LILIACEAE } \\
\hline Asphodelus albus & $35.800 \pm 7.454$ & 69,06 & $6,54 \pm 1,36$ & 12,61 & $4,28(0,33)$ \\
\hline Asphodelus ramosus & $24.000 \pm 3.816$ & - & $3,86 \pm 0,61$ & - & - \\
\hline Scilla autumnalis & $11.033 \pm 4.880$ & - & $0,57 \pm 0,25$ & - & - \\
\hline \multicolumn{6}{|l|}{ LINACEAE } \\
\hline Linum suffruticosum & $6.791 \pm 58$ & 25,65 & $0,91 \pm 0,01$ & 3,43 & $0,31(0,10)$ \\
\hline \multicolumn{5}{|l|}{ MALVACEAE } & $<0,01(<0,04)$ \\
\hline \multicolumn{6}{|l|}{ MYRTACEAE } \\
\hline \multicolumn{6}{|l|}{ OLEACEAE } \\
\hline Olea europaea var. oleaster & $137.775 \pm 9.476$ & $1.565,40$ & $1,41 \pm 0,09$ & 16,02 & $4,50(0,28)$ \\
\hline Olea europaea var. sylvestris & s $\quad 110.533 \pm 2.451$ & $1.037,02$ & $1,13 \pm 0,02$ & 10,6 & $3,14(0,30)$ \\
\hline \multicolumn{6}{|l|}{ OXALICACEAE } \\
\hline \multicolumn{6}{|l|}{ RAFFLESIACEAE } \\
\hline Cytinus hypocistis & $673.955 \pm 187.275$ & - & $2,61 \pm 0,72$ & - & - \\
\hline \multicolumn{6}{|l|}{ RANUNCULACEAE } \\
\hline $\begin{array}{l}\text { Anemone palmata } \\
\text { Ranunculus gramineus }\end{array}$ & $\begin{array}{c}121.517 \pm 30.005 \\
232.280 \pm 145.211\end{array}$ & $\begin{array}{c}96,73 \\
-\end{array}$ & $\begin{array}{l}3,87 \pm 0,95 \\
4,41 \pm 2,75\end{array}$ & 3,08 & $<0,01 \quad(<0,04)$ \\
\hline \multicolumn{6}{|l|}{ RESED ACEAE } \\
\hline Reseda suffruticosa & $396.813 \pm 52.886$ & - & $5,12 \pm 0,68$ & - & - \\
\hline \multicolumn{6}{|l|}{ RHAMNACEAE } \\
\hline Rhamnus velutinus (m) & $25.000 \pm 707$ & 513,97 & $0,14 \pm 0$ & 1,44 & $0,09(0,90)$ \\
\hline \multicolumn{6}{|l|}{ ROSACEAE } \\
\hline $\begin{array}{l}\text { Prunus persica } \\
\text { Prunus }\end{array}$ & $88.108 \pm 14.835$ & $\begin{array}{l}199,42 \\
217,45\end{array}$ & $0,88 \pm 0,14$ & $\begin{array}{l}0,39 \\
2,17\end{array}$ & $<0,01(<0,004)$ \\
\hline Rubus ulmifolius & $177.000 \pm 21.213$ & 590,47 & $1,74 \pm 0,20$ & 5,8 & $<0,01(<0,04)$ \\
\hline \multicolumn{6}{|l|}{ SMILACEAE } \\
\hline Smilax aspera (m) & $33.400 \pm 848$ & 86,3 & $0,28 \pm 0,01$ & 0,72 & $0,03(0,04)$ \\
\hline $\begin{array}{l}\text { VALERIANACEAE } \\
\text { Fedia cornucopiae }\end{array}$ & $5.066 \pm 2.082$ & 29,44 & $0,57 \pm 0,23$ & 3,31 & $<0,01(<0,04)$ \\
\hline
\end{tabular}

Tabla 1. Producción de polen de las especies estudiadas. $\mathbf{n}^{0} \mathbf{g P} / \mathbf{f l o r}: \mathrm{n}^{\circ}$ de granos de polen por flor; $\mathbf{n}^{0} \mathrm{gP} /$ $\mathrm{S}: \mathrm{n}^{\mathrm{o}}$ de granos de polen por $\mathrm{m}^{2}$ de superficie ocupada por una planta y durante un año (en millones); VgP/ flor: volumen de granos de polen por flor $\left(\mathrm{en}^{\mathrm{mm}} \mathrm{m}^{3}\right) ; \mathbf{V g P} / \mathbf{S}$ : volumen de granos de polen $\left(\mathrm{en}^{\mathrm{c}} \mathrm{cm}^{3}\right)$ por $\mathrm{m}^{2}$ de superficie ocupada por una planta y durante un año; IP: importancia polinífera en la Sierra de Mijas (=VgP/S x M\%); M\%: porcentaje de cobertura lineal en la sierra. (m): especies en las que se ha contado el número de granos de polen únicamente en flores masculinas. Pollen production of species studied. $\mathrm{n}^{\circ} \mathrm{gP} /$ flor: number of pollen grains per flower; $\mathbf{n}^{\circ} \mathrm{gP} / \mathbf{S}$ : number of pollen grains per $m^{2}$ of plant area and year (in millions); VgP/flor: volume of pollen grains per flower (in $\mathrm{mm}^{3}$ ); $\mathbf{V g P / S}$ : volume of pollen grains (in $\left.\mathrm{cm}^{3}\right)$ per $\mathrm{m}^{2}$ of plant area and year; IP: polliniferous importance in Sierra de Mijas $(=\mathrm{Vg} P / S \times M \%) ; \mathbf{M} \%$ : percentage of lineal cover in Sierra de Mijas. (m): male. 
mayor productividad floral (Hidalgo et al., 1996). Lo mismo ocurre si se comparan los $\mathrm{n}^{\circ} \mathrm{gP} / \mathrm{flor}$ y $\mathrm{n}^{\circ} \mathrm{gP} / \mathrm{S}$ de Lavandula stoechas y Teucrium fruticans; la segunda especie produce más número de granos de polen por flor que la primera, pero Lavandula stoechas produce más por unidad de superficie.

Los valores máximos de volumen de polen por flor ( $\mathrm{VgP} / \mathrm{fl}$ or) corresponden a Cistus albidus $\left(16,34 \mathrm{~mm}^{3}\right)$, Malva hispanica $(12,16$ $\left.\mathrm{mm}^{3}\right)$, Cistus crispus $\left(8,53 \mathrm{~mm}^{3}\right)$ y Cistus salvifolius $\left(7,19 \mathrm{~mm}^{3}\right)$; las especies de Cistus debido a su elevado número de granos de polen por flor y Malva hispanica debido al gran tamaño de sus granos de polen.

Respecto al volumen de polen por $\mathrm{m}^{2} \mathrm{y}$ año (VgP/S), los máximos corresponden a Lavandula stoechas $\left(125,46 \mathrm{~cm}^{3}\right)$, Quercus coccifera $\left(42,09 \mathrm{~cm}^{3}\right)$, Quercus rotundifolia $\left(24,26 \mathrm{~cm}^{3}\right)$, Ptilostemon hispanicus $\left(21,9 \mathrm{~cm}^{3}\right)$ y Cistus albidus $\left(17,7 \mathrm{~cm}^{3}\right)$. En los casos de Ptilostemon hispanicus y Cistus albidus se observa que se deben a sus elevados volumenes de polen por flor, mientras que en Quercus coccifera y Quercus rotundifolia se deben a sus alta producción de flores. Cistus crispus y Cistus salvifolius no tienen un volumen de polen por unidad de superficie alto porque su productividad floral es baja (Hidalgo et al.,
1996).

Las especies más importantes en producción de polen total (IP) en la zona de estudio, en función fundamentalmente de su cobertura, son Lavandula stoechas $(163,92)$, Rosmarinus officinalis (114,29), Cistus albidus (107,06), Quercus coccifera (74,17), Quercus rotundifolia (67,21), Cistus clusii $(22,59)$, Thymbra capitata $(18,72)$, Ulex baeticus $(15,44)$ y Cistus monspeliensis $(11,90)$. Otras especies, muy productivas en polen por flor y $\mathrm{m}^{2}$, presentan IP muy bajos dadas su escasa representación en la estructura del matorral estudiado.

Se ha representado gráficamente la distribución de frecuencia (en porcentaje de especies) para cuatro intervalos de producción de volumen de polen por flor (fig. 1A), volumen por superficie de planta (fig.1B) e IP (fig.1C). Se observa que hay un mayor porcentaje de especies $(59,02 \%)$ que presentan una producción de $\mathrm{Vgp} /$ flor baja $\left(\leq 1 \mathrm{~mm}^{3}\right)$. Sin embargo, con respecto a la producción por superficie de planta, ocurre lo contrario, la mayoría de las especies de la zona $(82,14 \%)$ tienen una producción alta $\left(\mathrm{Vgp} / \mathrm{S} \geq 1 \mathrm{~cm}^{3} / \mathrm{m}^{2}\right)$. En relación con el IP sólo nueve especies $(15,5 \%)$ presentan valores superiores a $10, y$ 23 especies $(43,3 \%)$ tienen valores superiores
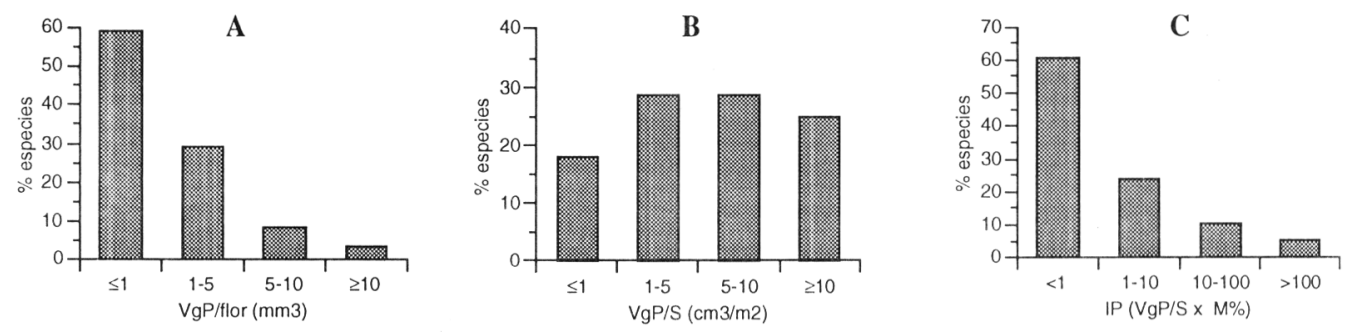

Figura 1. A, distribución de frecuencia de especies con diferente producción de volumen de polen por flor (VgP/flor). B, distribución de frecuencia de especies con diferente producción de volumen de polen por superficie de planta y fenofase floral completa $(\mathrm{VgP} / \mathrm{S})$. C, distribución de frecuencia de especies con diferente importancia polinífera en la Sierra de Mijas (IP).A, Frequency distribution of species with different production of volume of pollen per flower (VgP/flor). B, frequency distribution of species with diferrent production of volume of pollen per plant area and complete floral phenophase (VgP/S). C, frequency distribution of species with different polliniferous importancer in Sierra de Mijas (IP). 
a 1. Destacan las especies de las familias Labiadas y Cistáceas, con tres especies cada una entre las nueve más importantes. Si contabilizamos las 23 especies con IP superior a 1 , vemos que 15 de ellas $(65,2 \%)$ pertenecen a cuatro familias (Cistáceas, Labiadas, Fabáceas y Fagáceas).

\section{BIBLIOGRAFÍA}

BOLCHI, G. -1986/87- Observazioni qualitative sui bottini di polline di Apis mellifera $\mathrm{L}$. in frutteti. Bol. Zool. agr. Bachic., 19: 99-110.

CRUDEN, R.W. -1977- Polen-ovule ratios: A comparative indicator of breeding systems in flowering plants. Evolution, 31: 32-46.

DEVESA, J.A., J. ARROYO y J. HERRERA -1985Contribución al conocimiento de la biología floral del género Lavandula L. Anales Jard. Bot. Madrid, 42(1): 165-186.

HERRERA, J. -1985- Biología reproductiva del matorral de Doñana. Tesis Doctoral. Universidad de Sevilla.

HIDALGO, M.I. y B. CABEZUDO - 1994- Fenología y volumen de floración del matorral de la Sierra de Mijas (Málaga, S. España). Acta Bot. Malacitana, 19: 123-136.

HIDALGO, M.I., B. CABEZUDO y M. RECIO 1996- Producción floral en un matorral del Sur de España. Anales Jard. Bot. Madrid, 54(1): 547-553.

LOUVEAUX, J. -1958- Recherches sur la rècolte du pollen par les abeilles (Apis mellifera L.). Ann. Abeille, 1(3): 113-188.

NIETO, J.M., S. PÉREZ y B. CABEZUDO -1988Datos sobre la vegetación dolomitícola del sector Rondeño (Sierra de Mijas, Málaga, España). Lazaroa, 10: 35-46.

NIETO, J.M., A. PÉREZ LATORRE y B. CABEZUDO -1991- Biogeografía y series de vegetación de la provincia de Málaga (España). Acta Bot. Malacitana, 16(2): 417-436.

ORTIZ, P.L. -1985- Análisis polínico de mieles y celdillas de las Sierras del Sur de Córdoba (España). An. Asoc. Palinol. Leng. Esp., 2: 353360 .

ORTIZ, P.L. -1988- Estudio melitopalinológico en el Andévalo (Huelva). An. Asoc. Palinol. Leng. Esp., 4: 64-72.

ORTIZ, P.L. - 1991- Melitopalinología en Andalucía Occidental. Tesis Doctoral. Universidad de Sevilla.

ORTIZ, P.L. -1994- The Cistaceae as food resources for honey bees in SW Spain. J. Apic. Res., 33(3): 136-144.

ORTIZ, P.L., FERNÁNDEZ, I. \& M. MARTÍN CACAO -1990- Estudio melitopalinológico en la comarca de Aracena (Huelva). Lagascalia, 16(1): 61-76.

RICCI ARDELI, G. \& G. VORWOHL - 1979- Mieles monofloras en el Mediterráneo, documentado con ayuda de análisis microscópico de mieles. XXVII Congreso Internacional de Apicultura. Atenas. Ed. Apimondia. Bucarest, 1979, pp. 201-208.

TALA VERA, S., J. HERRERA, J. ARROYO, P.L. ORTIZ y J.A. DEVESA -1988- Estudio de la flora apícola de Andalucía Occidental. Lagascalia, 15 (extra): 567-591.

WEBER EL-GHOBARY, M.O. - 1984- Observation of flowering, pollen, nectar and pollen loads, due to Apis mellifera in some mediterranean plants. Les Colloques de e'INRA, 21: 245-250.

Aceptado para su publicación en Mayo de 1996

Dirección de los autores. Departamento de Biología Vegetal. Facultad de Ciencias. Universidad de Málaga. Apdo. 59. 29080, Málaga. Fax. (95)2131944. 